\title{
Competitive stochastic growth model for the 3D morphology of eutectic Si in Al-Si alloys
}

\author{
Gerd Gaiselmann $^{\mathrm{a}, *}$, Ole Stenzel ${ }^{\mathrm{a}, *}$, Anastasia Kruglova $^{\mathrm{b}}$, Frank Muecklich $^{\mathrm{b}}$, \\ Volker Schmidt ${ }^{\mathrm{a}}$ \\ ${ }^{a}$ Institute of Stochastics, Ulm University, 89069 Ulm, Germany \\ ${ }^{b}$ Department of Functional Materials, University of Saarland, 66123 Saarbrücken, Germany
}

\begin{abstract}
A competitive stochastic growth model is developed for the simulation of the 3D morphology of eutectic silicon in Al-Si alloys, which represents the colonies of pairwise disconnected $\mathrm{Si}$ corals in an $\mathrm{Al}$ matrix. The model is based on ideas from stochastic geometry and multivariate time series analysis. The 3D model is validated by comparing morphological characteristics computed for experimental 3D FIB-SEM data, and for realizations drawn from the model. Good agreement between the simulation model and the experimental image data is shown confirming the efficiency of using the competitive stochastic growth model for the generation of virtual eutectic silicon morphologies.
\end{abstract}

Keywords: Al-Si alloys, coral-like eutectic $\mathrm{Si}$, stochastic growth model, multivariate time series, FIB-SEM tomography, birth-and-death process

\section{Introduction}

A competitive stochastic growth model is developed for the simulation of the 3D morphology of eutectic silicon in $\mathrm{Si}-\mathrm{Al}$ alloys, where hypoeutectic $\mathrm{Al}-\mathrm{Si}$ alloys contain less than $12 \%$ of $\mathrm{Si}$ and present a two-phase material, consisting of primary $\alpha-\mathrm{Al}$ dendrites and $\mathrm{Al}-\mathrm{Si}$ eutectic [1]. The $\mathrm{Si}$ particles consist of pairwise disconnected $\mathrm{Si}$ corals in an $\mathrm{Al}$ matrix.

Combination of good castability and corrosion resistance of Al-Si alloys with good mechanical properties makes these alloys very attractive for applications in automotive industry. In $\mathrm{Al}-\mathrm{Si}$ alloys, there is a strong relationship between morphology of eutectic Si and their mechanical properties. Commercially used modification of the microstructure morphology with Sr addition changes the morphology of Si from a coarse plate-like into refined fibrous structure, significantly improving mechanical properties of the alloy, particularly tensile strength and elongation $[2,3,4,5,6]$. The results reported by Shin et al. [6] have shown

\footnotetext{
*Corresponding author: phone: +4973150 23590, fax: +497315023649, e-mail: gerd.gaiselmann@uni-ulm.de, ole.stenzel@uni-ulm.de
} 
that Sr-modification of $\mathrm{Al}-10.5 \mathrm{Si}-2.0 \mathrm{Cu}$ recycled alloy leads to an increase of the elongation by a factor of two (from 2.0 to $4.1 \%$ ) and tensile strength by more than $10 \%$ (from 209 to $237 \mathrm{MPa}$ ), which is reflected by a higher quality index of the material.

Stochastic models - in the context of materials science - can be used to elucidate the relationship between morphology and functional properties [7]. Such a design of virtual materials can be obtained by generating a broad range of virtual morphologies according to the stochastic model (using different values for the model parameters) and analyzing their functional properties by numerical calculations. Thus, it is possible to detect morphologies with improved materials properties using computer experiments. This reduces the amount of real experiments which are much more cost- and time-consuming.

In this paper, we present a stochastic model for the morphology of eutectic $\mathrm{Si}$ corals in Al-Si alloys. It is organized in a three-stage approach: In a first step, we introduce a model for single corals, where every single coral is represented by a connected system of line segments (which are dilated later). We use multivariate time series to accurately describe the complex spatial correlations of the branches within single corals $[8,9]$. Secondly, based on the single-coral model we present a competitive growth model which regulates the growth of neighboring corals according to a 'birth-and-death' process. More precisely, if two corals are competing for space, i.e. if the smallest Euclidean distance between two corals falls below a certain threshold, one coral will stop growing ('death') and the other one can continue to grow and expand in space ('birth'). The starting points for the competitive growth model are chosen according to an isotropic and stationary Matérn hardcore point process in 3D. In a third and final step, the aggregation of disconnected corals (i.e. aggregation of line segments) is dilated in $3 \mathrm{D}$ to match the volume fraction of Si. The stochastic model for Al-Si alloys is validated by comparing morphological characteristics computed for a 3D image gained by FIB-SEM tomography, and for realizations of the Al-Si model.

The paper is organized as follows. Section 2 introduces the material and describes imaging of Al-Si alloys. Section 3 establishes the 3D Al-Si model which is given in terms of a competitive growth model. Furthermore, model fitting and model validation are discussed. Section 4 summarizes the results and provides a short outlook regarding possible future research.

\section{Materials and imaging}

We have performed a tomographic reconstruction and analysis of morphological parameters (volume fraction, particle density, connectivity, etc.) for several samples of Al-Si alloys. Usually, an addition of 0,03 wt.\% of $\mathrm{Sr}$ is already enough to change an eutectic Si morphology [6]. The Al-Si7 alloy investigated in the present paper contains $150 \mathrm{ppm}$ of $\mathrm{Sr}$ and has typical morphological characteristics of this type of alloys. Thus, it can be considered as representative. The alloy is produced by directional solidification leading to a formation of the described coral-like structure of Si particles with major orientation in the direction 
of the temperature gradient. The main goal is to develop a flexible stochastic simulation model which allows to describe this kind of structural morphologies (coral like, directionally solidified). After having developed a model for a typical experimental structure, it can be used for the (virtual) generation of other similar structures by fitting the model parameters to another experimental data sets, which is described in Section 3.3. The reconstructed volume of modified eutectic and an example of unmodified eutectic are shown in Figure 1, where the stochastic model describes the modified eutectic Si corals.
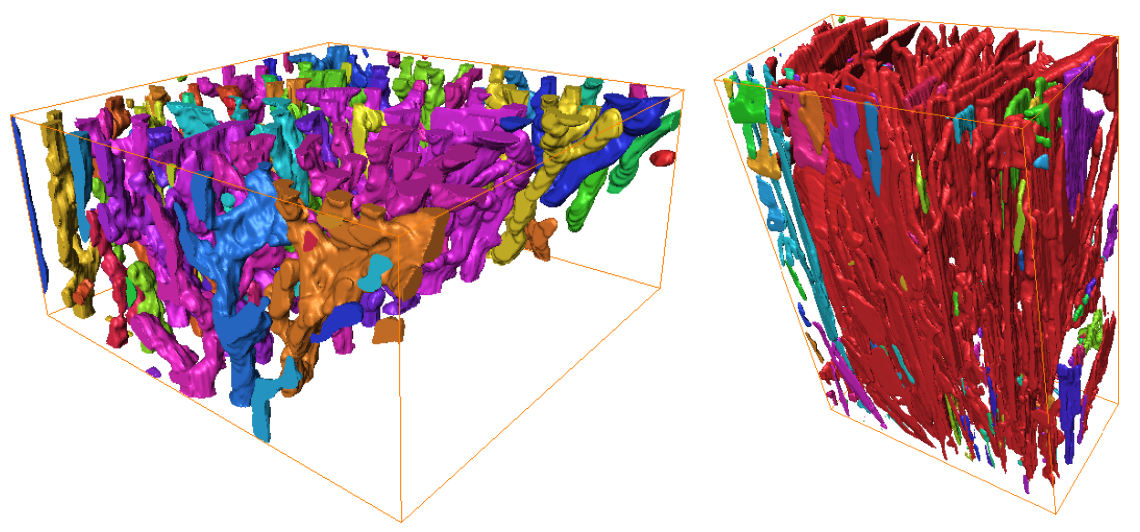

Figure 1: Left: 3D image of modified coral-like eutectic silicon; right: an example of unmodified lamellar or plate-like eutectic silicon; connected component are displayed by the same color

The reconstruction of the Al-Si eutectic has been done by using FIB-SEM dual beam tomography. The technique provides a high resolution of less than $50-60 \mathrm{~nm}[10]$ and allows imaging of microstructure morphology constituents with a good contrast. FIB-SEM tomography reconstruction consists of iterative milling of the sample with ion beam and imaging of the sectioned planes with electron beam after removing a slice of a certain thickness from the sample. Resolution in milling direction is defined as a thickness of the layer of material removed with an ion beam and depends on the precision of ion beam cuts. The resolution in the imaging plan depends on the resolution of SEM images. The angle between FIB and SEM columns is $52^{\circ}$, so that the sample surface is perpendicular and the imaging plan is parallel to the ion beam. Due to such an experimental setup the voxel of the reconstructed data volume is anisotropic and is bigger in the milling direction. For a detailed exposition of the technique see $[11,12]$. When a stack of 2D SEM images is collected, 3D reconstruction of the morphology is processed by interpolation in Avizo 6.3 software. The voxel resolution of the reconstructed $3 \mathrm{D}$ image is $46 \times 180 \times 59 \mathrm{~nm}$. The size of the considered image is $790 \times 195 \times 285$ voxel. 


\section{Stochastic model for Al-Si}

In this section, we introduce a stochastic model describing the morphology of eutectic Si in Al-Si alloys. Eutectic Si in this kind of material bears a coral-like structure, i.e., it consists of aggregates of $\mathrm{Si}$ corals which are pairwise disconnected, see Figure 1 (left). The stochastic model for the morphology of Si corals is organized in a three-stage approach, see also the flow chart in Figure 2.

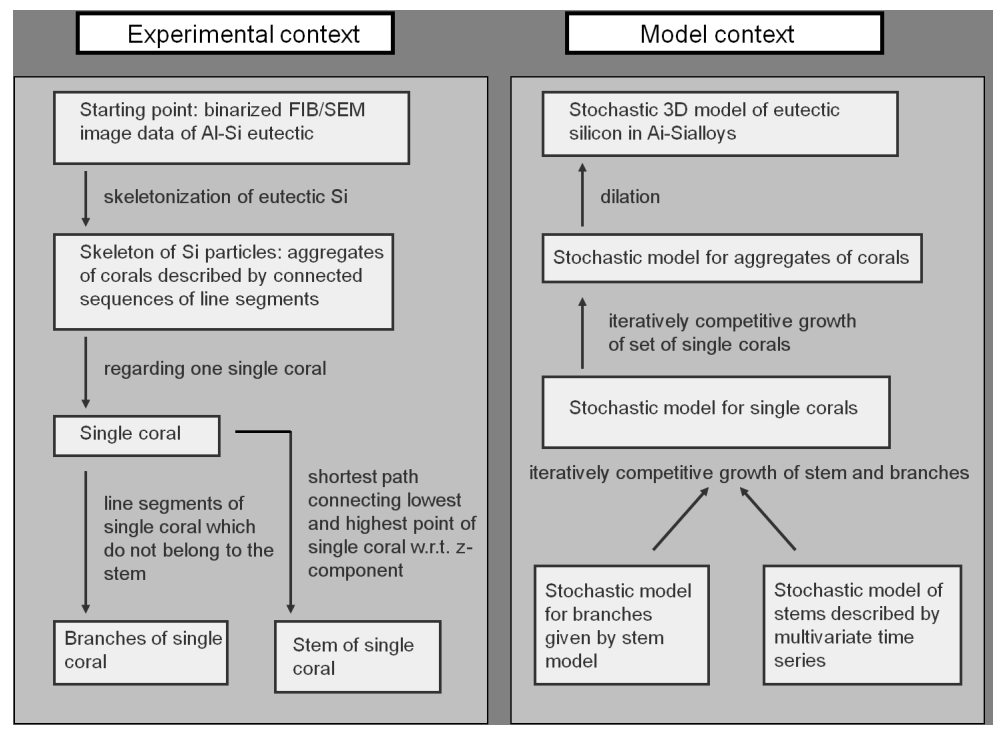

Figure 2: Flow chart of the stochastic model

In a first step, we introduce a model for single corals, where every single coral is represented by connected line segments, which form a stem and branches. Both components, the stem and the branches are modeled using multivariate time series to take into account the complex spatial correlations of the line segments within single corals. A 'win/lose' criterion is introduced to control the spatial expansion of the branches and the distances between neighboring branches.

Secondly, based on the single-coral model, a competitive growth model is introduced which regulates the growth of neighboring corals. We introduce some kind of a 'birth-and-death' process to control the distances between neighboring corals. More precisely, if two corals are competing for space, i.e., if the smallest Euclidean distance between two corals falls below a certain threshold, one coral will stop growing ('death') and the other one can continue to grow and expand in space ('birth'). In a third and final step, the aggregation of disconnected corals (i.e. aggregation of line segments) is dilated in 3D to match the volume fraction of $\mathrm{Si}$ as observed in the experimental image data. 


\subsection{Data preprocessing}

As described in Section 2, the FIB-SEM image of Al-Si alloys is given as binary image with anisotropic voxel. We consider a cut-out of the image and rescale the image to isotropic voxel using bilinear interpolation and subsequent global thresholding. The image size is $548 \times 761 \times 357$ (isotropic) voxel with voxel size of $46 \mathrm{~nm}$, see also Figure 3 (left). Thus, throughout the manuscript, we consider this 3D image of $\mathrm{Al}-\mathrm{Si}$ alloys with isotropic voxels. In order to describe the single corals by line segments whose evolvement is governed by a multivariate time series model, we reduce the morphology of Si to a graph representation consisting of nodes and edges (i.e. an aggregate of line segments). Therefore, the system of Si particles is skeletonized using Avizo 6.3 software, see Figure 3. This means that voxels of the $\mathrm{Si}$ particles, i.e., those voxels belonging to the objects we are interested in, are changed to background voxel in a way that the remaining voxel paths have a thickness of one voxel, where the connectivity has to be preserved. These voxel paths are then transformed into line segments, see also [13]. By this graph representation, we obtain accurate data on how to organize the spatial structure of the line segments.
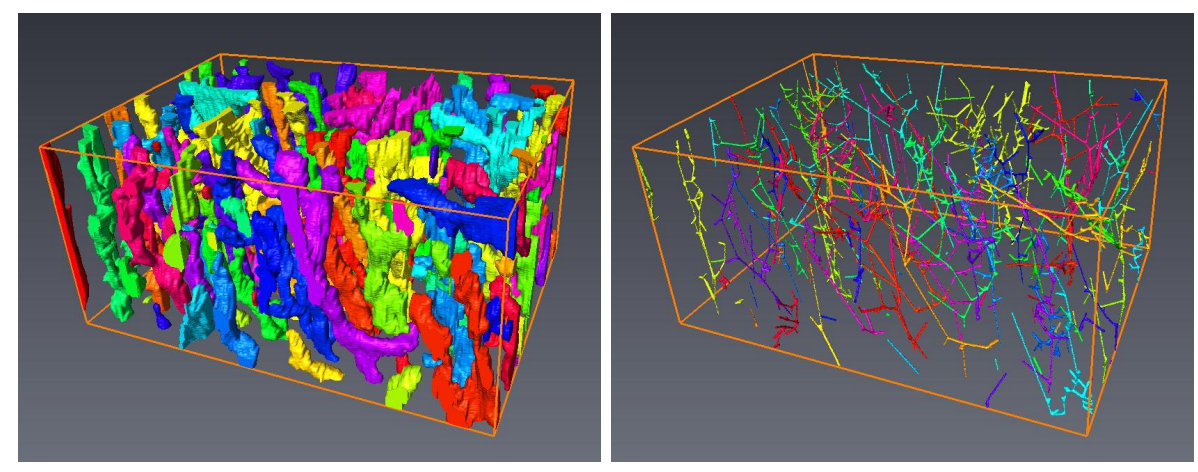

Figure 3: Left: 3D morphology of eutectic Si corals in an $\mathrm{Al}$ matrix; right: corresponding skeletonization; image size is $548 \times 761 \times 357$ voxel with voxel size of $46 \mathrm{~nm}$

\subsection{Model description}

The graph structure of the Si particles gained by skeletonization, as explained in the preceding section describes the main structure of the aggregation of $\mathrm{Si}$ corals. Based on this graph, we first introduce a stochastic model representing the graph structure of single corals. The single-coral model consists of two modeling components, the stem of a coral and its branches, where we define the stem of a coral by the shortest path connecting the lowest and highest point of a coral, see Figure 4. The advantage of this decomposition into stems and branches is that each component can be modeled using multivariate time series.

\subsubsection{Single-stem model}

In this section, we introduce a stochastic model for a stem as displayed in Figure 4 (right). The stem of a coral can be described as a polygonal track 

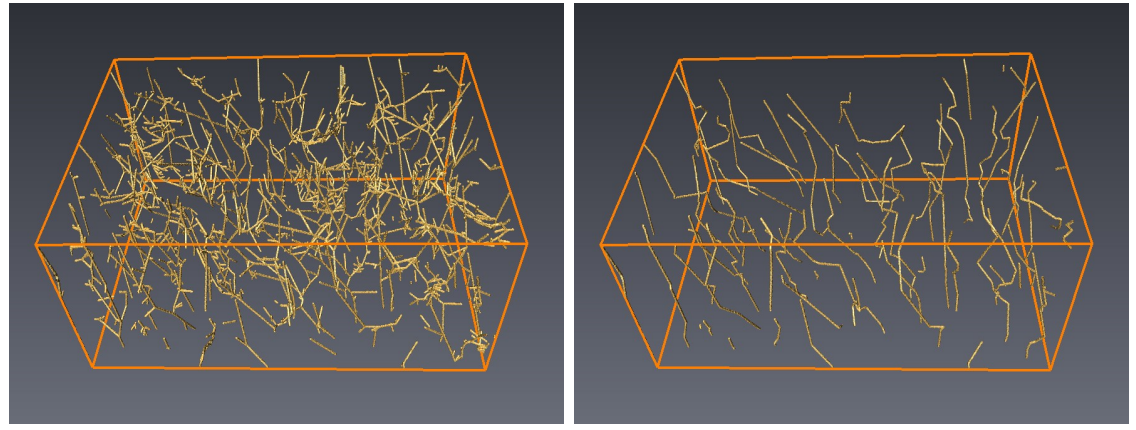

Figure 4: Left: 3D skeletonization of Si corals; right: corresponding stems

$p=\left(p_{1}, \ldots, p_{n}\right)$, where $p_{i}=\left(a_{i}, b_{i}\right)$ is the $i$-th line segment consisting of a starting point $a_{i} \in \mathbb{R}^{3}$ and an end point $b_{i} \in \mathbb{R}^{3}$.

The stem of a single coral will thus be modeled by a random polygonal track. This random polygonal track consists of a model for the first line segment (i.e. starting line segment) and a multivariate time series which describes the successive line segments, where the spatial correlations of consecutive line segments are taken into account by the time series.

Recently, this novel approach to describe polygonal tracks by multivariate times series has successfully been applied to model the courses of carbon fibers used in so-called gas-diffusion layers of proton exchange membrane fuel cells, see $[14,15]$.

To begin with, we state an alternative representation of polygonal tracks by the following incremental approach. Instead of describing a polygonal track $p=\left(p_{1}, \ldots, p_{n}\right)$ by the endpoints $a_{i}, b_{i} \in \mathbb{R}^{3}$ of its line segments $p_{i}$, we now consider an angle-length representation, where given the first line segment, the remaining line segments are described by the lengths $\ell_{1}, \ell_{2}, \ldots$ of the consecutive line segments and the angles $\alpha_{1}, \alpha_{2}, \ldots$ and $\beta_{1}, \beta_{2}, \ldots$. The angle $\alpha_{i}\left(\beta_{i}\right)$ denotes the change of direction from the $i$-th to the $(i+1)$-th segment with respect to the azimuthal (polar) angle. Note that the vector $\left(\alpha_{i}, \beta_{i}\right)$ is a point on the unit sphere. In summary, under the condition that the first line segment is given, a polygonal track is uniquely described by the sequence of vectors $\left(\alpha_{1}, \beta_{1}, \ell_{1}\right)^{\top},\left(\alpha_{2}, \beta_{2}, \ell_{2}\right)^{\top}, \ldots$, see Figure 5 .

Thus, it would be reasonable to describe the polygonal tracks given in this incremental representation, by a 3D multivariate time series. However, looking at cross-correlations, see Figure 5 (right), we see that the first component, i.e., the azimuth angle $\alpha$, seems to be (stochastically) independent of the polar angle $\beta$ and of the length of the line segment $\ell$. Moreover, it seems that consecutive azimuth angles are also independent from each other. Therefore, we model the sequence of azimuth angles by a sequence of independent and normally distributed random variables $\alpha_{1}, \alpha_{2}, \ldots$, i.e., $\alpha_{n} \sim N\left(\mu, \sigma^{2}\right)$. The remaining two components, i.e., the polar angle and the length of the line segment, do have crosscorrelations, see Figure 5 (right). Hence, the sequence of consecutive vectors 

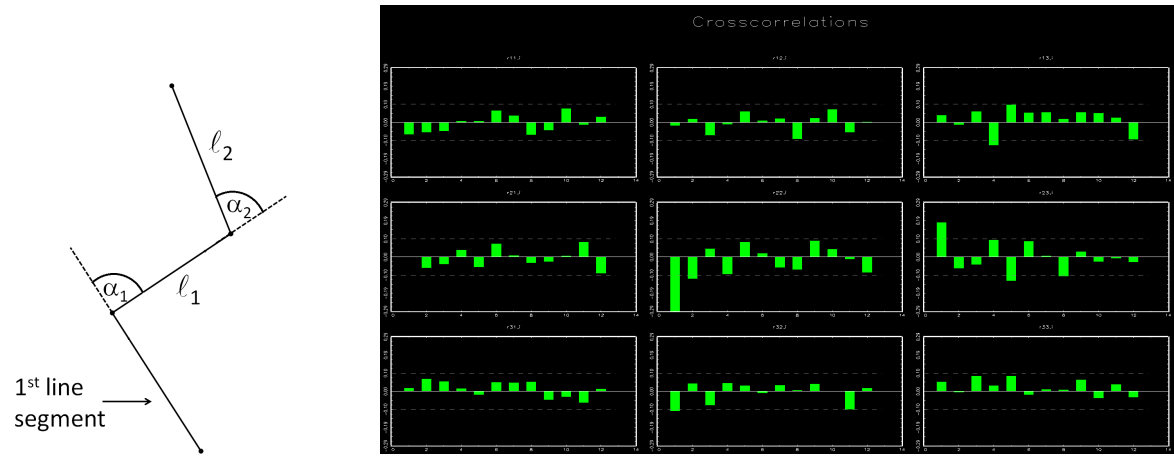

Figure 5: Left: incremental representation of polygonal tracks in the 2-dimensional case; right: cross-correlation of $\left\{G_{i}\right\}$

$\left(\beta_{i}, \ell_{i}\right)$ of polar angles and lengths of line segments is modeled by a $2 \mathrm{D}$ multivariate time series $\left\{Y_{i}\right\}$, which will be specified later. Thus, the single-stem model is described by the $3 \mathrm{D}$ stochastic process $\left\{G_{i}\right\}=\left\{\Psi\left(\alpha_{1}, Y_{1}\right), \Psi\left(\alpha_{2}, Y_{2}\right), \ldots\right\}$, where the function $\Psi: \mathbb{R}^{3} \rightarrow \mathbb{R}^{3}$ is given by

$$
\begin{aligned}
& \Psi(r, s, t)=\left(r-2 k_{1} \pi, s-2 k_{2} \pi, \max \{0, t\}\right), \\
& \text { if }\left(2 k_{1}-1\right) \pi \leq r<\left(2 k_{1}+1\right) \pi \text { and }\left(2 k_{2}-1\right) \pi \leq s<\left(2 k_{2}+1\right) \pi
\end{aligned}
$$

for some $k_{1}, k_{2} \in \mathbb{Z}$, where $\mathbb{Z}=\{\ldots,-1,0,1, \ldots\}$ denotes the set of integers, and the definition of $\Psi$ assures some natural regularity, i.e., the changes of directions of consecutive line segments are not allowed to be larger than $\pi$, the lengths of line segments have to be non-negative. The geometric interpretation of the process $\left\{G_{i}, i \geq 1\right\}$ is that its first (second) component is a sequence of random angles denoting the change of direction from the $i$-th to the $(i+1)$-th line segment of a polygonal track w.r.t. the azimuthal (polar) angle. The third component specifies the lengths of the consecutive line segments of a polygonal track.

In order to fully describe the stem of single corals, we consider as starting line segment $\ell_{\text {start }}$ a vertical line segment with a length $\ell$ which is inverse Gaussian distributed, i.e., $\ell \sim \operatorname{inv} G\left(\mu_{1}, \mu_{2}\right)$ with density

$$
f_{\mu_{1}, \mu_{2}}(x)=\left(\mu_{2} /\left(2 \pi x^{3}\right)\right)^{1 / 2} \exp \left(\left(-\mu_{2}\left(x-\mu_{1}\right)^{2}\right) /\left(2 \mu_{1}^{2} x\right)\right) .
$$

In summary, the stochastic model $\left\{X_{i}, i \geq 1\right\}$ for the stem of corals is given by $\left\{X_{i}\right\}=\left(\ell_{\text {start }},\left\{G_{i}\right\}\right)$.

Finally, the time series $\left\{Y_{i}, i \geq 1\right\}$ is chosen by means of an autoregressive process of order 2. That means that $\left(\beta_{i}, \ell_{i}\right)$ is a function of its two previous values $\left(\beta_{i-1}, \ell_{i-1}\right),\left(\beta_{i-2}, \ell_{i-2}\right)$ plus some random component. More precisely, the autoregressive processes $\left\{Y_{i}, i \geq 1\right\}$ of order 2 is given by

$$
Y_{i}=\eta+A_{1} Y_{i-1}+A_{2} Y_{i-2}+\varepsilon_{i} \quad \text { for each } i \geq 1 \text {. }
$$


Note that $\eta \in \mathbb{R}^{2}$, and the coefficient matrices $A_{1}, A_{2} \in \mathbb{R}^{2 \times 2}$ are considered as model parameters. The 'residuals' $\left\{\varepsilon_{i}, i \geq 1\right\}$ are assumed to form a sequence of two-dimensional random vectors which are independent and identically distributed with vanishing mean vector $\mathbb{E} \varepsilon_{i}=o$ and some (non-singular) covariance matrix $\Sigma=\mathbb{E}\left(\varepsilon_{i} \varepsilon_{i}^{\top}\right)$ where $\Sigma$ is a further model parameter, see e.g. [8, 9]. For more information about random polygonal track models based on multivariate time series, the reader is referred to $[14,15]$.

Parameter Estimation. The parameters $\eta, A_{1}, A_{2}, \Sigma$ are estimated as described in detail in [14]. In our case, we get that

$$
Y_{i}=\left(\begin{array}{c}
-0.65 \\
1.34
\end{array}\right)+\left(\begin{array}{cc}
-0.45 & 0.41 \\
-0.02 & -0.004
\end{array}\right) Y_{i-1}+\left(\begin{array}{cc}
-0.25 & 0.07 \\
0.01 & 0.015
\end{array}\right) Y_{i-2}+\varepsilon_{i},
$$

where $\varepsilon_{i} \sim \mathrm{N}\left(\left(\begin{array}{l}0 \\ 0\end{array}\right),\left(\begin{array}{cc}0.17 & -0.01 \\ -0.01 & 0.01\end{array}\right)\right)$. Moreover, the first component (polar angle) of $\left\{Y_{i}, i \in \mathbb{Z}\right\}$ is a dimensionless number whereby the second component (length of line segments) is given with respect to the cubic voxel size of the rescaled (experimental) 3D FIB-SEM data which is equal to $46 \mathrm{~nm}^{3}$. A transformation of this voxel size can be easily obtained by multiplying all $Y_{i 2}$ with factor $\frac{x}{46}$, where $x$ is the desired new voxel size. Regarding the estimation of $\left(\mu, \sigma^{2}\right)$, we consider the usual maximum-likelihood estimator applied to the sample of angles $\alpha_{i}$ denoting the change of direction from the $i$-th to the $(i+1)$ th line segment w.r.t. the azimuthal angle of the extracted stems of corals. For the parameter $\left(\mu_{1}, \mu_{2}\right)$ of the inverse Gaussian distribution, representing the length of the starting line segments, we consider the maximum-likelihood estimator applied to the sample of all line segments which are observed in the graph structure of the Al-Si material. As results, we obtain $\left(\mu, \sigma^{2}\right)=(0.025,1.5)$ and $\left(\mu_{1}, \mu_{2}\right)=(1.16,1.29)$. The values for $\left(\mu_{1}, \mu_{2}\right)$ are given in $\mu m$.

\subsubsection{Stochastic modeling of single corals}

In this section, we develop a model to add branches to the single stems introduced in Section 3.2.1, which yields the single-coral model. When analyzing the graphs extracted from the experimental Si corals, it turns out that at the end of each line segment of the extracted stem, there exists one branch. Therefore, we include branching to the stem model by including exactly one branch at the end of each line segment. The branch itself is simulated according to the stem model (i.e. with same time series model which is used for the stem), but with a different starting line segment, which will be specified later on.

Obviously, different branches may interact or even cross each other. To control the interaction of branches or the distances between branches, respectively, we introduce a 'win/lose' criterion, which we specify in the following. The general idea is that all line segments from different branches should have a reasonable distance from each other. First we simulate a single stem and number serially the endpoints of its line segments starting at 1 , see Figure $6(0)$. Then, in the first iteration step, we add a new branch at the endpoint labeled with 1 (Figure 6 (1)). In the $i$-th iteration step for $i \geq 1$, we add a new branch at 
all endpoints of the existing line segments which have the mark $i$, see Figure 6 (2). At the end of each iteration step, we (eventually) delete new line segments according to the following rules, see Figure 6 (3).

- All line segments from the stem and the first line segment of a new branch located at the stem 'survive'.

- If the smallest Euclidean distance between a candidate for new line segment $p=(a, b)$ and any other line segment (excluding the new ones) is below a threshold $\tau_{\text {internal }}$, the new line segment is deleted ('lose'). However, if the distance from the starting point $a$ (to the nearest line segment) is smaller than at the endpoint $b$, i.e., the new branch is moving away from the remaining part of the tree, the line segment is not deleted ('win'). This exception is useful since branches with common branching point are rather close, still have a chance to 'survive'.

- If the smallest Euclidean distance between a new line segment and another new line segment is below a threshold $\tau_{\text {internal }}$, the line segment with the highest end point (i.e. $z$-coordinate) 'wins' and the other 'loses' (i.e. is deleted), see Figure 6 (3). However, if the distance from the starting point $a$ (to the nearest line segment) is smaller than at the endpoint $b$, i.e., the new branch is moving away from the remaining part of the tree, the line segment is not deleted ('win'). Again, this exception is useful since branches with common branching point are rather close, still have a chance to 'survive'.

- The thinning is organized in such a way that we start with the line segment which has the smallest $z$-component. Then, the deletion takes place iteratively, i.e., the order in which line segments are deleted, does have an influence on the final result.

To complete the single-coral model, we have to specify the choice of the starting line segment for the branches. Our intention is that all branches have the same azimuth angle $( \pm \pi)$, in agreement with the Si corals observed in the experimental data, see Figure 7 (left). Therefore, we mark each coral with an angle $\delta$, where $\delta$ is an uniformly distributed random variable in the interval $[0, \pi]$, i.e., $\delta \sim U(0, \pi)$. The starting line segment $\ell_{\text {branch }}=(a, b)$ of the branch is uniquely described by its starting and end points $a, b$, where the starting point is already given. Using spherical coordinates, the endpoint $b$ is described by its azimuth angle $\varphi$, polar angle $\theta$ and length $\ell$, where $\varphi$ is uniformly distributed on $[\delta-\pi, \delta+\pi]$. The polar angle $\theta$ is chosen in dependence of the previous line segment $\ell_{\text {prev }}$ where the branch begins, see Figure 7 (right). More precisely, $\theta$ is determined by an acceptance and rejection method. We choose $\theta$ as a realization of a uniformly distributed random variable in the interval $[-\pi, \pi]$ and we accept $\theta$ if the angle between $\ell_{\text {branch }}$ and the previous line segment $\ell_{\text {prev }}$ is in the interval $\left[\frac{\pi}{5}, \frac{4 \pi}{5}\right]$, otherwise we sample a new realization of $\theta$. 
(0)

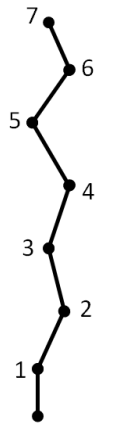

(1)

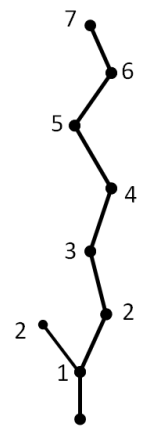

(2)

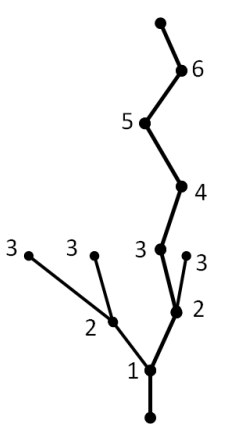

(3)

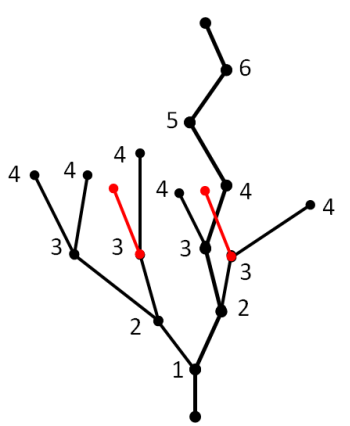

Figure 6: Modeling idea of single coral: First the main stem is described by a random polygonal track where the endpoints of these line segments are numbered serially $(0)$. Branches are added to the stem and numbered serially $((1),(2))$ and finally branches are deleted $((3)$, red colored) which are too close to each other
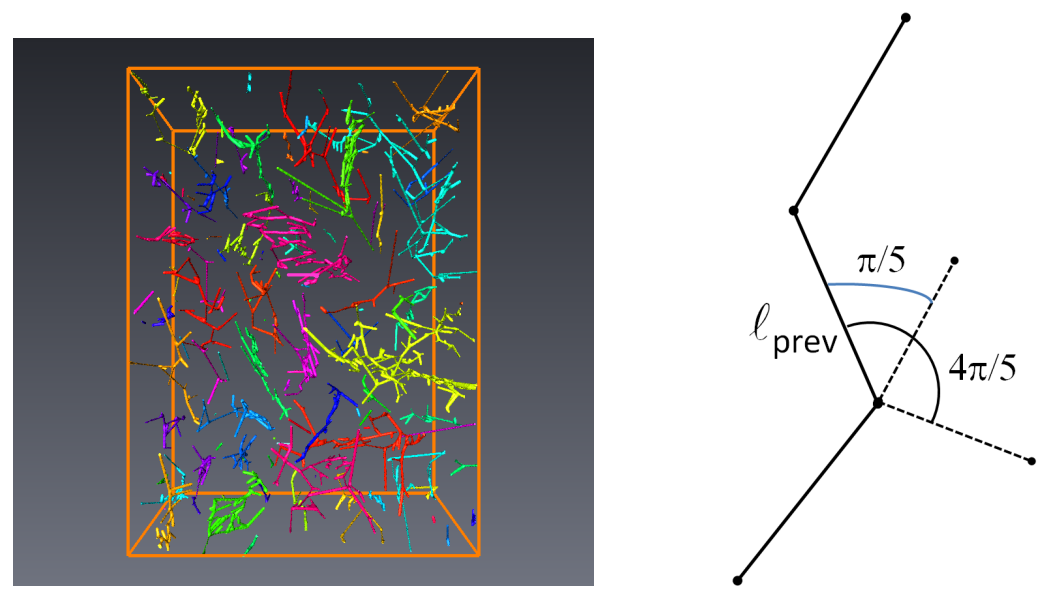

Figure 7: Left: top view on graph of Si corals; right: illustration of choice of polar angle for new branch 


\subsubsection{Stochastic modeling of aggregates of corals}

Based on the single-coral model introduced in Sections 3.2.1 and 3.2.2, we develop a stochastic model of aggregates of corals whose realizations correspond to the graph structure of Si corals as displayed in Figure 3. This is done by a two-stage growing approach.

In the first stage, single corals grow iteratively as described in Section 3.2.2, where the starting points are given by a stationary and isotropic point process in $3 \mathrm{D}$. More precisely, as a stochastic model representing the random starting points of the single corals, we choose a Matérn hardcore process with some intensity $\lambda$ and hardcore radius $r_{h c}>0$ in the sampling window $\mathbb{R} \times \mathbb{R} \times[0, \infty)$, see [16]. Note that in a Matérn hardcore process, all points have a minimum distance $r_{h c}>0$ from each other. This is an important aspect to ensure that the Si corals are nicely spread in space and do not interfere directly at the beginning of their growing process. We let all corals grow simultaneously at their starting points given by the point process. During the growth process, we propose some kind of 'birth-and-death' process to control the distances between neighboring single corals. It is obvious that single corals must not touch each other (to ensure the correct number of separated single corals), but we also want to control the distances between them. Therefore, the 'birth-and-death' process is organized as follows:

- If branches belonging to two different corals are too close to each other, i.e., the minimal distance of these branches (of stems) is smaller than $\tau_{\text {external, }}$ the growth of that branch (stem) which has the smaller $z$-component stops growing ('death').

- In each iteration step of the growth process, the external competition criterion (given by $\tau_{\text {external }}$ ), being more important, is executed before the internal competition criterion (given by $\left.\tau_{\text {internal }}\right)$. Thereby, it is possible that (segments of) stems are deleted (and only the branches continue to grow).

- The thinning is organized in such a way that we start with the line segment which has the smallest $z$-component. The deletion takes place iteratively, i.e., the order in which line segments are deleted, does have an influence on the final result.

We denote the union of those corals by $\Xi^{(0)}$. The 'birth-and-death' process of the first stage gives nice control of the distances between neighboring corals. However, as a side-effect, the 'birth-and-death' process yields large pores, i.e. regions with a large shortest distance to the nearest $\mathrm{Si}$ coral. This is plausible since the winning single coral ('birth') needs some time to take over the free space of the other single coral. The branches of the winning single coral will expand in space, but it will not directly take over the free space.

Therefore, in the second stage, these 'gaps' are filled by an iterative Cox procedure. The idea is to identify large 'gaps' and let corals grow starting from their midpoints. In the first iteration step, we simulate corals in the 'gaps' of 
the plane $P_{0}=\mathbb{R} \times \mathbb{R} \times\left\{z_{0}\right\}$ with $z_{0}=0$, i.e., we start at the bottom of our observation window. We aim to let the new corals start growing in the centers of the 'gaps' from the plane $P_{0}$. Therefore, we determine the local maxima in the plane $P_{0}$ of the so-called distance transformation of $\Xi^{(0)}$ which assigns to each point $x \in \mathbb{R} \times \mathbb{R} \times[0, \infty)$ the shortest Euclidean distance to the nearest coral and denote it by $D_{\Xi^{(0)}}(x)$. To avoid that new corals interfere with other corals of the first stage, the set of local maxima is thinned out by first deleting each local maximum $m$ such that $D_{\Xi^{(0)}}(m)<r_{c o x}$. Thus, every new coral has a minimum distance to a coral from the first stage larger than $r_{c o x}$. Secondly, if the Euclidean distance of two local maxima $m_{1}, m_{2}$ is smaller than $r_{\text {cox }}$, we delete $m_{1}$ if $D_{\Xi^{0}}\left(m_{1}\right)<D_{\Xi^{0}}\left(m_{2}\right)$, and $m_{2}$ otherwise. This ensures that two new corals have a minimum distance (at least at their first line segments) of $r_{c o x}$ such that they do not interfere directly. Now, we let corals grow starting from the remaining local maxima of the plane $P_{0}$ according to the same procedure as used for corals in the first stage while taking into account these already existing corals. More precisely, if during the growth process of these new corals the distances of some of their branches to the already existing corals, here $\Xi^{(0)}$, are smaller than $\tau_{\text {external }}$, these branches are deleted. Otherwise, if the distances of branches of different new corals are smaller than $\tau_{\text {external, }}$, then the growth of that branch which has a smaller $z$-component stops. We denote the union of the new corals by $\Xi^{(1)}$. This procedure is repeated iteratively, where in the $i$-th iteration step, we consider the plane $P_{i}=\mathbb{R} \times \mathbb{R} \times\left\{z_{i}\right\}$ with $z_{i}=(i-1) \frac{r_{c o x}}{2}$.

Investigations accomplished by means of realizations of this model for aggregates of corals have shown that these realizations reach a steady-state in the sampling window $\mathbb{R} \times \mathbb{R} \times[100, \infty)$, where the dimension is given in voxel (100 voxel correspond to $4.6 \mu \mathrm{m}$ ). This process towards a steady state can be explained as follows, where we begin by the first stage: corals start growing according to a realization of a 3D Matérn hardcore process, which is stationary and isotropic. Those corals that start at the bottom, i.e. with $z$-component close to zero, will have plenty of space to develop branches. Sooner or later, however, every coral will interfere with other corals (whose starting point may be far above) and stop growing. Those corals that started at the bottom, may thus have a larger maximum stem length. This bias will decreases with increasing $z$-component. For large $z$-components, the process will eventually reach an equilibrium. This shows that the first stage of the competitive stochastic growth model reaches stationarity (for large $z$ ). Similarly, the iterative Cox procedure also leads to stationarity for sufficiently large $z$ for the same reasons.

Thus, for that reason our model is defined on the (slightly smaller) sampling window $\mathbb{R} \times \mathbb{R} \times[200, \infty)$, such that the process in $\mathbb{R} \times \mathbb{R} \times[0,200)$ can reach its equilibrium.

Parameter Estimation. The hardcore radius $r_{h c}$ is chosen such that $\lambda$ stands for the maximal possible intensity with the hardcore radius $r_{h c}$ of a hardcore process, i.e, $r_{h c}=\left(\frac{3.0}{4 \pi \lambda}\right)^{\frac{1}{3}}$. The estimation of the remaining parameters $\lambda, \tau_{\text {external }}, r_{c o x}$ is described in Section 3.3. A realization of the stochastic model for aggregates of corals is displayed in Figure 8. 

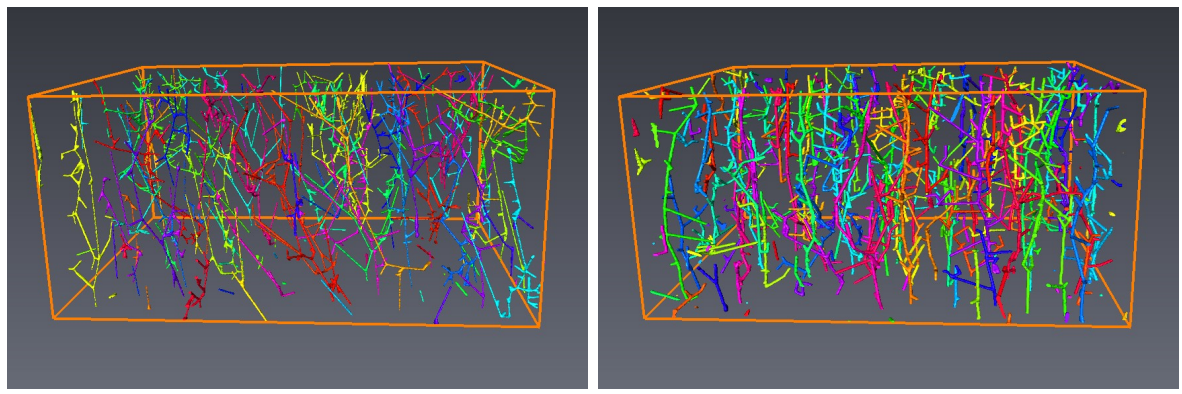

Figure 8: Left: 3D graph structure of $\mathrm{Si}$ corals in an $\mathrm{Al}$ matrix; right: realization of the stochastic model for aggregates of corals

\subsubsection{From graph structure to morphology}

The stochastic model for aggregates of corals introduced in Section 3.2.3 describes the graph structure of eutectic Si corals and consists of an ensemble of line segments. From a mathematical point of view, the volume of this arrangement of line segments is equal to zero. Therefore, in this final modeling step, we transform the graph onto a 3D grid and dilate the graph such that a) the volume fraction of Si corals is matched and that b) separate corals remain separated after dilation. Therefore, we separately dilate each edge of the aggregates of corals model with a sphere as structuring element and radius $r_{\text {dilate }}>0$. Now, the radii of edges which overlap with edges of other corals are simultaneously decreased by some small value $\varepsilon>0$ until the dilated edges from different corals show no more overlap. The radius $r_{\text {dilate }}>0$ is chosen such that the volume fraction after the iterative erosion is matched.

\subsection{Model fitting}

The stochastic model presented in this work is fully parameterized. Most parameters could be estimated in a straightforward way. The remaining parameters for the internal and external competition, $\tau_{\text {internal }}, \tau_{\text {external }}$, and the parameters for the starting points $\lambda, r_{c o x}$ are estimated by the so-called minimum contrast method. Therefore, aggregates of $\mathrm{Si}$ corals are simulated in dependence of the parameter vector $v=\left(\tau_{\text {internal }}, \tau_{\text {external }}, \lambda, r_{c o x}\right)$. The value of $v$ for which the discrepancy between structural characteristics of simulated $\mathrm{Si}$ corals and their experimental counterparts is minimized, is called a minimum contrast estimator. More precisely, we aim to minimize the cost function

$$
\begin{aligned}
L(v)= & \int_{b_{11}}^{b_{12}}\left|H(r)-H_{v}(r)\right| d r+\int_{b_{21}}^{b_{22}}\left|E L(r)-E L_{v}(r)\right| d r \\
& +\int_{b_{31}}^{b_{32}}\left|T L(r)-T L_{v}(r)\right| d r .
\end{aligned}
$$

Here, $H, H_{v}\left(E L, E L_{v} ; T L, T L_{v}\right)$ denote the spherical contact distribution function (distribution of edge length; distribution of maximum stem length), 
computed for the graph structure of the experimental $\mathrm{Si}$ corals and for realizations of the multi-coral model, respectively. More precisely, the value $H(r)$ of the spherical contact distribution function denotes the probability that the minimum distance from a randomly chosen location of the $\mathrm{Al}$ phase to the graph structure is not larger than $r$ for $r>0$. Furthermore, the distribution of maximum stem length is defined by the distribution of lengths of shortest paths connecting the lowest and highest point of the corals. The constants $b_{11}, b_{12}, b_{21}, b_{22}, b_{31}, b_{32}$ are appropriately chosen integration limits. A vector $v_{0}$ such that $L\left(v_{0}\right) \leq L(v)$ holds for all admissible values of $v$ is called a minimum contrast estimator for $v=\left(\tau_{\text {internal }}, \tau_{\text {external }}, \lambda, r_{c o x}\right)$. In Figure 9 we can clearly see that the three structural characteristics used for the minimum-contrast estimation are almost perfect represented by our growth model.
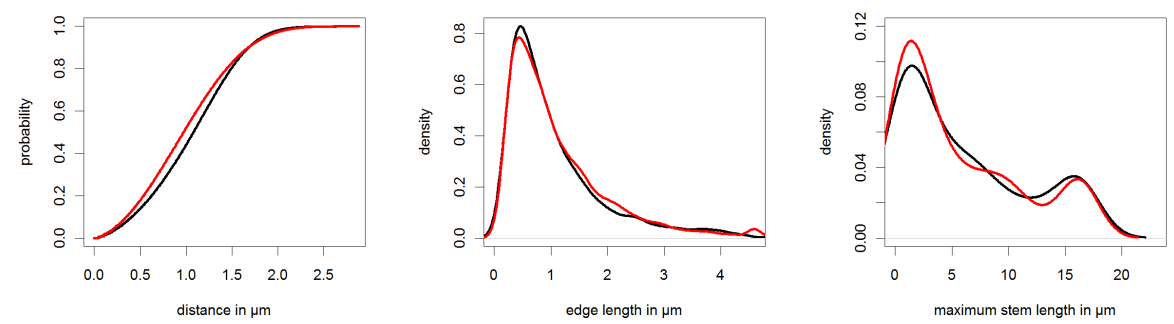

Figure 9: Left: spherical contact distribution function; center: distribution of edge lengths; right: distribution of maximum stem length; black: computed for the graph structure of experimental Si corals, red: drawn from the multi-coral model

Finally, the radius $r_{\text {dilate }}$ for the dilation of line segments is chosen such that after the simultaneous decrease of radii, the volume fraction of the Si structure is fitted. The numerical values of the fitted parameters are given by $\tau_{\text {internal }}=$ $1.38 \mu m, \tau_{\text {external }}=0.92 \mu m, \lambda=1.34 e-7, r_{h c}=5.47 \mu m, r_{c o x}=2.53 \mu m$ and $r_{\text {dilate }}=0.55 \mu \mathrm{m}$.

\subsection{Influence of model parameters}

In the previous section, we estimated the model parameters that describe the structure of Al-Si alloys. For reasons of clarity, in Figure 10, all model parameters are listed and their influence is shortly described. In the following, we will discuss the influence of model parameters on the microstructure of Al$\mathrm{Si}$ alloys in more detail. This is also an important aspect regarding virtual materials design in order to generate different virtual microstructures of $\mathrm{Al}-\mathrm{Si}$ alloys. We begin with the parameters for the stochastic model for the single corals. Mainly, the evolution and correlation structure of the connected system of line segments is described by the parameters of the time series model including parameters for the starting line segment. The degree of branching, however, is controlled by the 'internal competition' parameter $\tau_{\text {internal, }}$, where, roughly speaking, $\tau_{\text {internal }}$ is the maximum distance which is required between branches 


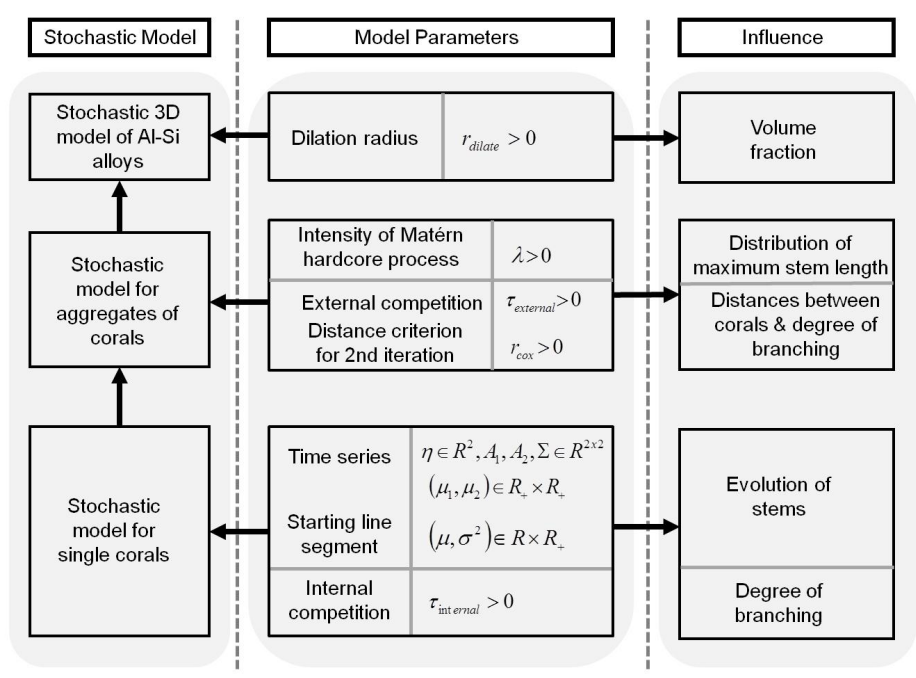

Figure 10: Overview of model parameters and their influence on virtual microstructures of Al-Si alloys

belonging to the same coral. A lower value of $\tau_{\text {internal }}$ leads to a significant increase in the number of branches as fewer branches are deleted according to the internal criterion as described in Section 3.2.2. Similarly, the 'external competition' parameter $\tau_{\text {external }}$ as well as $r_{\text {cox }}$ control the size of corals and the distance between them, where a higher value of $\tau_{\text {external }}\left(r_{c o x}\right)$ reduces the average distance between separate corals while producing larger corals. The intensity of the Matérn hardcore process $\lambda$ controls the relative frequency of corals with a very large maximum stem length. Suppose $\lambda$ is low, then there are few starting points in 3D for the corals to grow. Thus, in the first stage of the competitive growth algorithm, there is less interference with other corals which means that these corals have a large maximum stem length. Finally, the dilation radius $r_{\text {dilate }}$ influences the volume fraction of the corals, where a larger radius leads to a larger volume fraction.

\subsection{Model validation}

In this section, we want to elucidate to which extend the stochastic simulation model agrees with the experimentally determined Si corals. In Figure 11 (right) a typical realization of the Al-Si model is shown. By visual inspection, the simulated image coincides quite well with the experimental FIB-SEM image which is shown on the left-hand side of Figure 11.

In the following, we check more formally if the 3D stochastic model describes the morphology of the Al-Si material sufficiently well.

Therefore, the stochastic simulation model will be validated by comparing morphological characteristics computed for the 3D image gained by FIB-SEM 

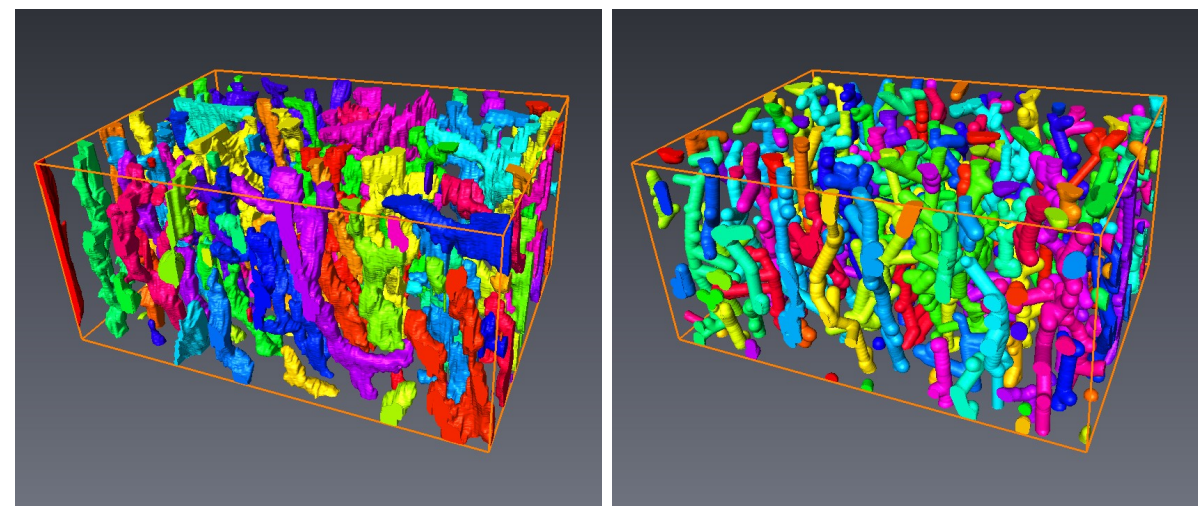

Figure 11: Left: 3D morphology of experimental Si corals in an $\mathrm{Al}$ matrix; right: corresponding simulation of stochastic model; image size is $761 \times 548 \times 357$ voxel with voxel size of $46 \mathrm{~nm}$

Table 1: Basic morphological characteristics

\begin{tabular}{cccccc|}
\hline & $V_{v}$ & $\begin{array}{c}S_{v} \\
{\left[\mu m^{-1}\right]}\end{array}$ & $\begin{array}{c}M_{v} \\
{\left[\mu m^{-2}\right]}\end{array}$ & $\begin{array}{c}K_{v} \\
{\left[\mu m^{-3}\right]}\end{array}$ & $\begin{array}{c}\text { particle density } \\
{\left[\mu m^{-3}\right]}\end{array}$ \\
\hline real & 0.14 & $5.27 \mathrm{E}-1$ & $3.84 \mathrm{E}-1$ & $6.03 \mathrm{E}-3$ & $1.04 \mathrm{E}-2$ \\
\hline simulated & 0.13 & $4.95 \mathrm{E}-1$ & $4.80 \mathrm{E}-1$ & $5.84 \mathrm{E}-2$ & $1.32 \mathrm{E}-2$ \\
\hline
\end{tabular}

tomography, and for realizations of the Al-Si model, respectively.

\subsubsection{Comparison of densities}

In materials science, there are four basic parameters used for the characterization of morphologies in 3D. These parameters are also called 'densities' since they are determined relative to the sample volume. The definitions and methods for the estimation of these characteristics, including stereological ones, are discussed in [17] and [18]. Below we briefly discuss general properties of these basic parameters. The volume density $V_{v}$ provides information about material constitution. The surface density $S_{v}$ reflects many of materials' properties such as mechanical strength, dispersion, etc. A typical example to demonstrate an influence of surface density on mechanical properties of a material is the Hall-Petch relationship. It relates the surface density of the $\mathrm{Si}$ phase with the material strength. The specific integral of mean curvature $M_{v}$ depends on the geometrical characteristics of the $\mathrm{Si}$ phase, e.g. precipitates, and is related to the deformation behavior of materials. The specific integral of total curvature $K_{v}$ is directly related to the mean number of particles per unit volume that is, in turn, defined by the nucleation rate.

Agreement between the described parameters for both experimental and simulated morphologies is particularly important for admitting a model as an adequate one. Table 1 presents comparative results of morphology analysis for the stochastic model and tomography-reconstructed 3D image. As one can see, 
there is a satisfying agreement between results for volume fraction $V_{v}$, specific surface area $S_{v}$ and specific integral of mean curvature $M_{v}$.

The values of specific integral of total curvature differ by one order of magnitude. The reason for such a difference may be that the integral of total curvature $K_{v}$ is sensitive to the density of particles, where the particle density is slightly overestimated by the stochastic model. Besides, the estimation of $K_{v}$ may be unstable.

\subsubsection{Distributional characteristics}

Furthermore, we consider the distribution of spherical contact distances from $\mathrm{Al}$ to $\mathrm{Si}$ particles, and vice versa, where also a good agreement is found, see Figure 12. Note that we already considered this characteristic for model fitting of the aggregates of corals (which is a system of line segments), but now we calculate this characteristic for the complete 3D microstructure of Al-Si alloys. Next, the distribution of chord-lengths in the directions of $x-, y-$ and $z-$ axes is determined. Figure 13 shows the cumulative distribution function of the chordlength in each of these three directions. One can see clearly that there is a good accordance w.r.t. to these chord-lengths distribution. Overall, we can conclude a good agreement of the stochastic model with the aggregate of experimentally determined Si corals.
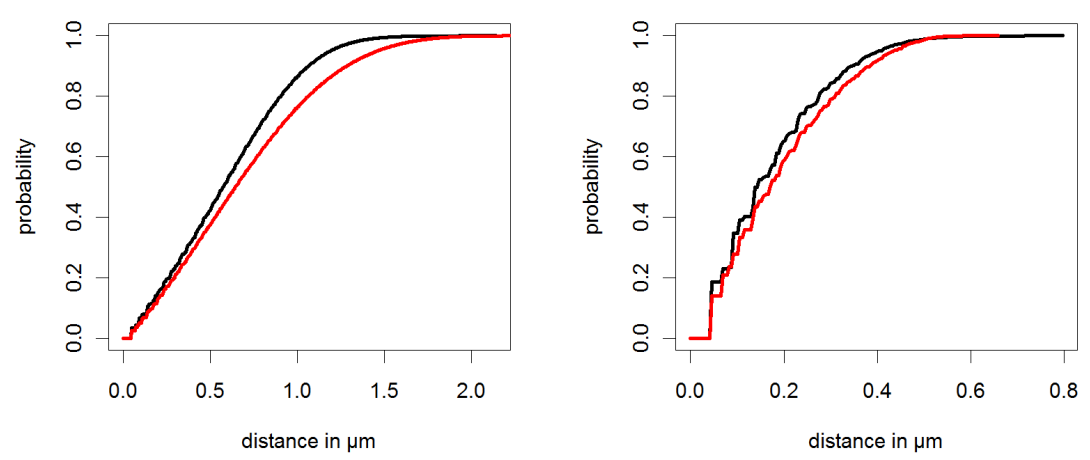

Figure 12: Distribution of spherical contact distances from $\mathrm{Al}$ to Si particles (left), and vice versa (right) for the experimental image data (black curve) and realization drawn from the stochastic model (red curve)

\section{Conclusions and Outlook}

We have proposed a stochastic 3D model for simulating the spatial morphology of eutectic silicon in Al-Si alloys. This fully parametrized model is based on ideas from stochastic geometry and multivariate time series analysis. The 

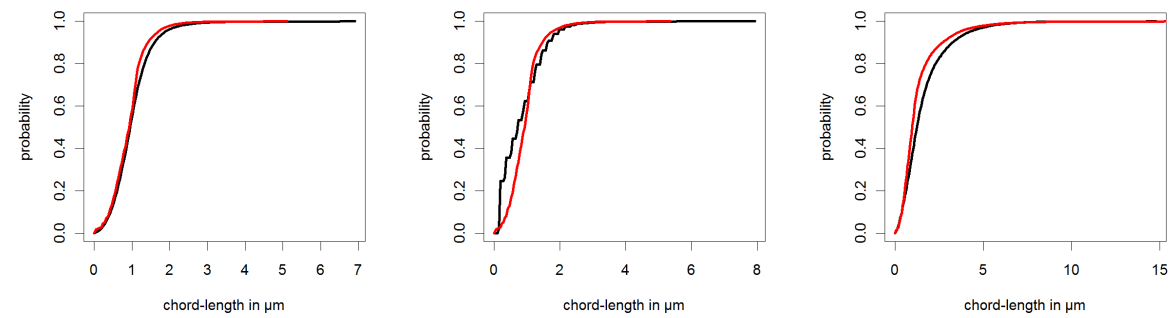

Figure 13: Distribution of chord lengths in direction $x-$ (left), $y-$ (center) and $z-$ (right) axes for the experimental image data (black curve) and realization drawn from the stochastic model (red curve)

model is organized in a three-stage approach: In a first step, a model for single corals is introduced, where every single coral is represented by connected line segments (which are dilated later). Multivariate time series are used to accurately describe the complex spatial correlations of the branches within single corals. Second, based on the single-coral model, we present a competitive growth model which regulates the growth of neighboring corals according to a certain 'birth-and-death' process. The final step is a dilation of the set of trees (corals) in order to fit the volume fraction of silicon. The stochastic model is validated by comparing morphological parameters computed for a $3 \mathrm{D}$ tomographic image and for realizations of the model. Although some differences are discovered for the density of corals, good agreement of the other parameters is shown, confirming the model as adequate for generation of virtual eutectic silicon morphologies. Further investigations are required to study the flexibility of the model with respect to various structural scenarios. In particular, our model will be used for virtual scenario analyses in a forthcoming work with the general aim to detect a correlation between the microstructure morphology and its mechanical properties. An optimal eutectic Si structure providing the best mechanical properties can be deduced by systematic modifications of the model parameters and in combination with mechanical simulations.

\section{References}

[1] ASM International Handbook Committee (eds.) (1990). Properties and Selection: Nonferrous Alloys and Special-Purpose Materials. ASM Handbook, Vol. 2, ASM International.

[2] Closset, B., Gruzleski, J.E. (1982). Structure and properties of hypoeutectic $\mathrm{Al}-\mathrm{Si}-\mathrm{Mg}$ alloys modified with pure strontium. Metallurgical and Materials Transactions A, 13, 945-951a.

[3] Haque, M.M. (1995). Effects of strontium on the structure and properties 
of aluminium-silicon alloys. Journal of Materials Processing Technology, 55, 193-198.

[4] Hess, P.D., Blackmun, E.V. (1975). Strontium as a modifying agent for hypoeutectic aluminum-silicon alloys. AFS Transactions, 83, 87-90.

[5] Lasagni, F., Lasagni, A., Holzapfel, C., Mücklich, F. and Degischer, H.P. (2006). Three dimensional characterization of unmodified and Sr-modified Al-Si eutectics by FIB and FIB EDX tomography. Advanced Engineering Materials, 8, 719-723.

[6] Shin, S.S., Kim, E.S., Yeom, G.Y., Lee, J.C. (2012). Modification effect of $\mathrm{Sr}$ on the microstructures and mechanical properties of $\mathrm{Al}-10.5 \mathrm{Si}-2.0 \mathrm{Cu}$ recycled alloy for die casting. Materials Science and Engineering A, 532, 151-157.

[7] Stenzel, O., Koster, L.J.A., Oosterhout, S.D., Janssen, R.A.J., and Schmidt, V. (2012). A new approach to model-based simulation of disordered polymer blend solar cells. Advanced Functional Materials, 22, 1236-1244.

[8] Fuller, W.A. (1996). Introduction to Statistical Time Series. 2. ed., New York: J. Wiley \& Sons.

[9] Lütkepohl, H. (2006). New Introduction to Multiple Time Series Analysis. Berlin: Springer.

[10] Lasagni, F., Lasagni, A., Engstler, M., Degischer, H.P. and Mücklich, F. (2008). Nano-characterization of cast structures by FIB-tomography. Advanced Engineering Materials, 10, 62-66.

[11] Giannuzzi, L.A. and Stevie, F.A. (2005). Introduction to Focused Ion Beams. New York: Springer.

[12] Holzer, L., Indutnyi, F., Gasser, P.H., Münch, B. and Wegmann, M. (2004). Three-dimensional analysis of porous $\mathrm{BaTiO}_{3}$ ceramics using FIB nanotomography. Journal of Microscopy, 216, 84-95.

[13] Thiedmann, R. Hartnig, C., Manke, I., Schmidt, V. and Lehnert, W.. (2009). Local structural characteristics of pore space in GDL's of PEM fuel cells based on geometric 3D graphs. Journal of the Electrochemical Society, 156, B1339-B1347.

[14] Gaiselmann, G., Thiedmann, R., Manke, I., Lehnert, W. and Schmidt, V. (2012). Stochastic 3D modeling of fiber-based materials. Computational Materials Science, 59, 75-86.

[15] Gaiselmann, G., Froning, D., Manke, I., Lehnert, W. and Schmidt, V. (2012). Stochastic 3D modeling of non-woven GDL with wet-proofing agent. Preprint (submitted). 
[16] Illian, J., Penttinen, A., Stoyan, H. and Stoyan, D. (2008). Statistical Analysis and Modelling of Spatial Point Patterns. Chichester: J. Wiley \& Sons.

[17] Ohser, J. and Mücklich, F. (2000). Statistical Analysis of Microstructures in Materials Science. Chichester: J. Wiley \& Sons.

[18] Russ, J., C. and Dehoff, R., T. (2000). Practical Stereology. New York: Kluwer Academic/Plenum Publishers. 